\title{
Method of Hardware Selection of Characteristic Features Based on Radon Transformation and not Sensitive to Rotation, Shifting and Scale of the Input Images
}

\author{
Stepan Bilan ${ }^{1}$, Ruslan Motornyuk ${ }^{2}$, Sergii Bilan $^{3}$ \\ ${ }^{1}$ Faculty "Infrastructure and rolling stock of railways", State Economy and Technology University \\ of Transport, Kiev, Ukraine \\ ${ }^{2}$ Data Processing Center of Southwestern Railways, Kyiv, Ukraine \\ ${ }^{3}$ Win-Interactive LLC, Vinnytsia, Ukraine \\ ${ }^{1}$ bstepan@ukr.net, ${ }^{2}$ xehap0@gmail.com, ${ }^{3}$ belan@svitonline.com
}

\begin{abstract}
In this paper considered by the method of the organization of cellular automata to extract characteristic features of images based on the Radon transform. Cellular automata are constructed using a hexagonal surface. This increases the efficiency of image recognition. This made it possible to implement the Radon transform on the six directions. This cellular automata was simulated in the software environment of Active-HDL, and also was created his software model. Experimental studies for both models showed high results of recognition for the different levels of noise that are present in the image.
\end{abstract}

Keywords: Cellular automata, Radon transform, Image recognition, Hexagonal coverage.

\section{Introduction}

In 1917, the mathematician J. Radon [1,2] proposed a method of renewal (reconstruction) of multidimensional functions by their integral characteristics. The method solves the inverse problem of integral geometry. Besides of the main applications of the Radon transform (RT) (computed tomography $[3,4]$, seismology) there are papers which prove its effectiveness on the allocation of characteristic features such as image recognition tasks [5] and at the analysis of their structure [1,5-9].

On the basis of RT we can build a system for automatic recognition of visual images. Shortcomings in the existing fields of application are:

1) - medical tomographs implement this method through hardware, though relatively quickly, but not used for image of task recognition; 
2) - with digital imaging the entire volume calculations of Radon transform (which has a direct relationship with the Fourier transform [3], and hence a similar computational complexity), falls on CPU, which can not provide the performance for real-time.

Medical tomographs are stationary bulky devices that can not be effectively used anywhere, especially for the problem of recognition.

In this paper we consider the possibility of combining of hardware implementation of projections RT with small size of device. Hexagonal cells is used for organization of cellular automaton (CA), which provides high accuracy in image recognition and speed comparable to that of real-time [10-17]. RT is considered in six directions through the implementation of CA, based on cells with a hexagonal shape.

\section{Implementation of RT model on the CA with a hexagonal coated}

In [3] it is determined that a sufficient minimum number of RT projections, which is possible to restore primary image is three: the directions $0^{\circ}, 60^{\circ}$ and $120^{\circ}$. Hence a set of three projections can be regarded as the characteristic feature that is already possible to perform image recognition. But the above-mentioned angles $\left(0^{\circ}, 60^{\circ}\right.$ and $\left.120^{\circ}\right)$ exactly coincide with the directions of the hexagonal coverage (fig. 1,a) [15,17]. Moreover, the hexagonal lattice is possible to allocate not only 3 but and 6 lines (fig. 1,б). As shown in Figure 1,a is a cellular automaton in the form of a regular hexagon shape repeats its view of the hexagonal unit pixel. The same figure shows the lines of the main directions of the hexagonal coverage - is $0^{\circ}, 60^{\circ}$ and $120^{\circ}$. Besides the main directions of hexagonal raster has additional directions corresponding corners $30^{\circ}, 90^{\circ}$ and $150^{\circ}$. All these directions and their relative positions are shown in Figure 1,b, hence it is in these areas hexagonal raster enables constructing six projections RT (fig. 1,b). Hence, if you imagine the processed image as a homogeneous structure or matrix having a hexagonal surface (Fig. 1,a) and give it some rules of functioning, you can get a result in the form of six projections on directions RT $0^{\circ}, 30^{\circ}, 60^{\circ}, 90^{\circ}, 120^{\circ}, 150^{\circ}$ (fig. 1,b).

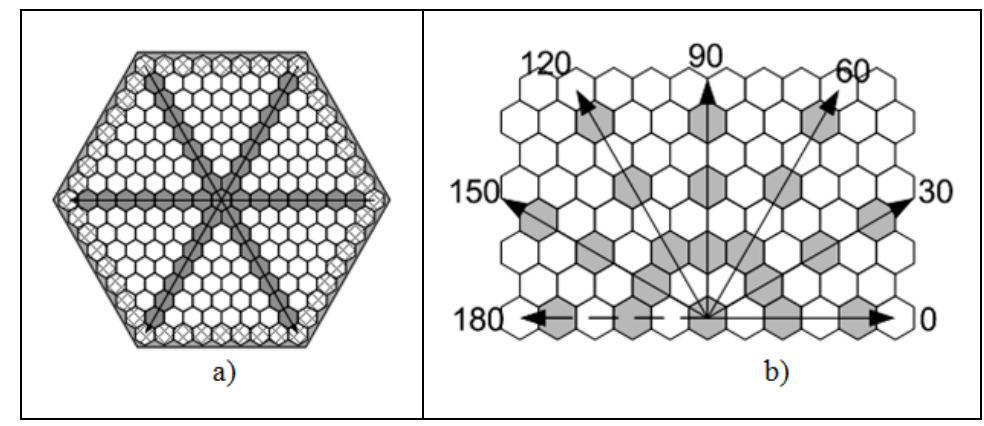

Figure 1: «Congenital» directions of hexagonal coverage

Such homogeneous structures have long been invented and are called CA. The main interest of the specialists in the field of computer science to cellular automata is caused by the fact that 
they form a parallel computing paradigm, just as Turing machine paradigm successive forms. Thus, they can be used as models which own natural parallelism [10-18].

Consider the Radon transform and its physical content in more detail.

The most important practical and at the same time the simplest case is a function of two variables.

Suppose we are given a function $f(x, y)$, which is defined on some interval $D[3,4]$. Consider in the plane $x y$ the line $L$, which intersect area $D$ (fig. 2). Then, by integrating the function $f(x, y)$ along the line $L$, we obtain the projection or linear integral of functions $f$. Integration along all possible lines $L$ allows to define the RT for the function $f(x, y)$ :

$$
\stackrel{\vee}{f}=R f=\int_{L} f(x, y) d s
$$

where ds - increment of length along the line L.

As is known [1-3] RT function $f(x, y)$ for the two-dimensional case through the normal equation of the line $L$ can be represented by a formula (fig. 2 ):

$$
\checkmark(p, \varphi)=R f=\int_{-\infty}^{\infty} f(p \cos \varphi-s \sin \varphi, p \sin \varphi+s \cos \varphi) d s
$$

where $p$-distance from the origin to the line (fig. 2);

$\varphi$ - the angle between the $p$ and $x$.

$d s$ - length of increment along the line L.

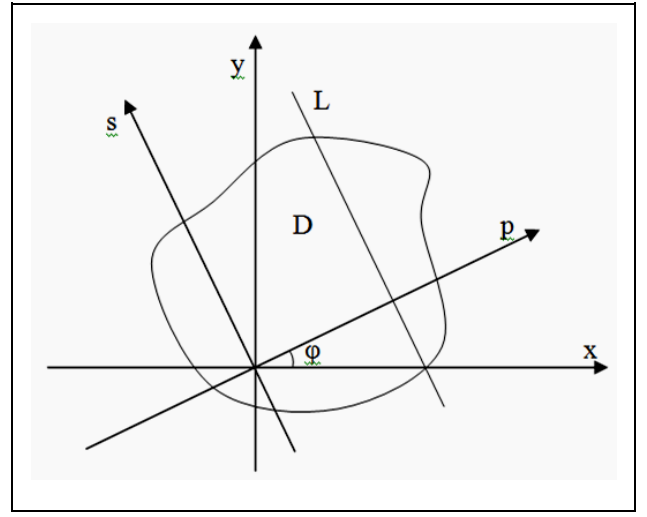

Figure 2: Radon transform

In a cellular automaton with a hexagonal surface (CAHS), which is built by hardware of six projections (sinograms) RT for directions $0^{\circ}, 30^{\circ}, 60^{\circ}, 90^{\circ}, 120^{\circ}$ and $150^{\circ}$ particular cases of the formula (2) will have the form:

For direction $0^{\circ}$ : 


$$
\stackrel{\vee}{f}_{0}(p, 0)=R_{0} f=\int_{-\infty}^{\infty} f(p, s) d s
$$

For direction $30^{\circ}$ :

$$
\stackrel{v}{f}_{30}(p, 30)=R_{30} f=\int_{-\infty}^{\infty} f\left(\frac{\sqrt{3}}{2} p-\frac{1}{2} s, \frac{1}{2} p+\frac{\sqrt{3}}{2} s\right) d s
$$

For direction $60^{\circ}$ :

$$
\tilde{f}_{60}(p, 60)=R_{60} f=\int_{-\infty}^{\infty} f\left(\frac{1}{2} p-\frac{\sqrt{3}}{2} s, \frac{\sqrt{3}}{2} p+\frac{1}{2} s\right) d s
$$

For direction $90^{\circ}$ :

$$
\tilde{f_{90}}(p, 90)=R_{90} f=\int_{-\infty}^{\infty} f(s, p) d s
$$

For direction $120^{\circ}$ :

$$
f_{120}^{v}(p, 120)=R_{120} f=\int_{-\infty}^{\infty} f\left(-\frac{1}{2} p-\frac{\sqrt{3}}{2} s, \frac{\sqrt{3}}{2} p-\frac{1}{2} s\right) d s
$$

For direction $150^{\circ}$ :

$$
f_{150}^{v}(p, 150)=R_{150} f=\int_{-\infty}^{\infty} f\left(-\frac{\sqrt{3}}{2} p-\frac{1}{2} s, \frac{1}{2} p-\frac{\sqrt{3}}{2} s\right) d s
$$

If necessary, a larger number of projections RT, which is allocated by hardware, can be used by two or more CAHS which are rotated relative by to each other by an angle $\beta$ to axis perpendicular to their plane:

$$
\angle \beta=30 / n
$$

where $n-$ is CAHS number, each of which performs a selection of RT by 6 directions.

Physical content of RT consists of finding of the sum of the brightness values of pixels, which forming this image, along a straight line in a certain direction. The results of these transformations will be arrays of values, which will transform the projections in the relevant directions.

Now we use the physical is content of the RT for set rules of operation of CA. To construct the projection sufficient to perform shift of the entire image in the desired direction. Here it is necessary to perform the summation of the luminances of pixels, which are not in the cells themselves, but in special accumulation adders which are situated on the perimeter of the cell medium.

Building of projection for angles $0^{\circ}, 60^{\circ}, 120^{\circ}$ is quite easy to implement. It is necessary to implement a shift of the entire image in the appropriate directions, typical for this type of 
coating. The fig. 3,b shows an example of a direction of shift $0^{\circ}$ : each cell $(x 0)$ takes the value of its right neighbor (x4). Similarly projections for directions $60^{\circ}$ and $120^{\circ}$ are constructed.

Projections for angles of $30^{\circ}, 90^{\circ}, 150^{\circ}$ are more complex to build and implement. The difficulty is that it is impossible to perform a shift along the marked directions. To get the desired angles, it is necessary to combine the shift in the neighboring directions in turns by $0^{\circ}$, $60^{\circ}, 120^{\circ}$. Let us consider the example of shift for the angle $30^{\circ}$. When moving left and down, each cell $(x 0)$ takes by turns the value of its upper right neighbor $(x 2)$ and right neighbor $(x 4)$ (fig. 3,c).

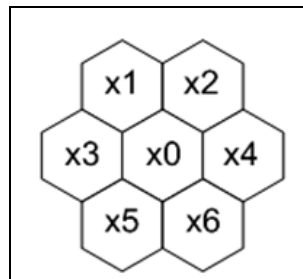

a)

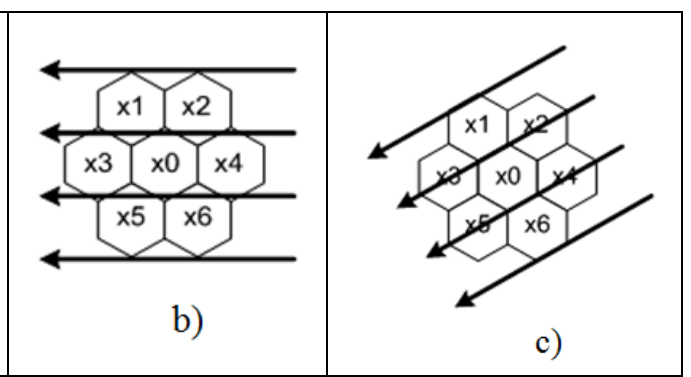

Figure 3: Codes of neighboring cells and shift of the image on the hexagonal cover

Considering all the above, we can describe the principle of operation of the device. Let $Q$ set of directions CAHS: $Q=\left\{0^{\circ}, 30^{\circ}, 60^{\circ}, 90^{\circ}, 120^{\circ}, 150^{\circ}\right\}$. If every line of the $i$-th $(i=\{1,2, \ldots$, $k\}$, where $k$ - the maximum length of the line) in the direction of $q$ imagine how many pixels $L_{q i}$, and such that $L_{q i}=\left\{x_{1}, \ldots, x_{k}\right\}$, then You can write the formula amount of luminance values of all pixels in the line

$$
\mathrm{S}_{\mathrm{qi}}=\sum_{j=1}^{\mathrm{Z}_{\mathrm{i}}} \mathrm{x}_{\mathrm{j}}
$$

where $Z_{i}$ - the maximum number of pixels in the $i$-th line. Then projection RT for direction q can be represented as an ordered set $R_{q}$, consisting of all sums $S_{q i}$ this direction: $R_{q}=\left\{S_{q 1}, S_{q 2}, \ldots\right.$, $\left.\mathrm{S}_{\mathrm{qk},}\right\}$.

Accordingly, a single processing element (PE) of CAHS $x 0$ at the current time $t(x 0(t))$ should be set to one of the neighboring PE $(x 1, x 2, x 3, x 4)$ in the preceding time $t-1\left(x 1_{(t-1)}, x 2_{(t-1)}, x 3_{(t-1)}\right.$, $\left.\mathrm{x} 4_{(\mathrm{t}-1)}\right)$ under the influence of appropriate control signals $(\mathrm{SX} 1, \mathrm{SX} 2, \mathrm{SX} 3, \mathrm{SX} 4): \mathrm{x} 0_{(\mathrm{t})}=\mathrm{SX} 1 \cdot \mathrm{x} 1_{(\mathrm{t}-1)}{ }^{+}$ $\mathrm{SX} 2 \cdot \times 2_{(\mathrm{t}-1)}+\mathrm{SX} 3 \cdot \times 3_{(\mathrm{t}-1)}+\mathrm{SX} 4 \cdot \times 4_{(\mathrm{t}-1)}$.

In work [15], a comparison of the program models and VHDL-models is performed, and also the analysis of the received projections is carried out. The experiment confirmed the similarity of the projections built during software modeling and Active-HDL models. It is results confirm the possibility of a hardware implementation of RT.

Calculations show that for processing of image with size of $8192 \times 8192$ pixels (67 megapixels) the cell medium will already spend 0,074 seconds at a frequency $1 \mathrm{MHz}$. That is, 
the performance is sufficient for selection of the characteristic features (CF) on the basis of the $\mathrm{RT}$ to build recognition systems that can operate in real time.

Examples of RT for image of letters «A» and 木 for different fonts and noise levels (Fig. 4a) show that this method of selection characteristic features that is resistant to insignificant distortions and noise.

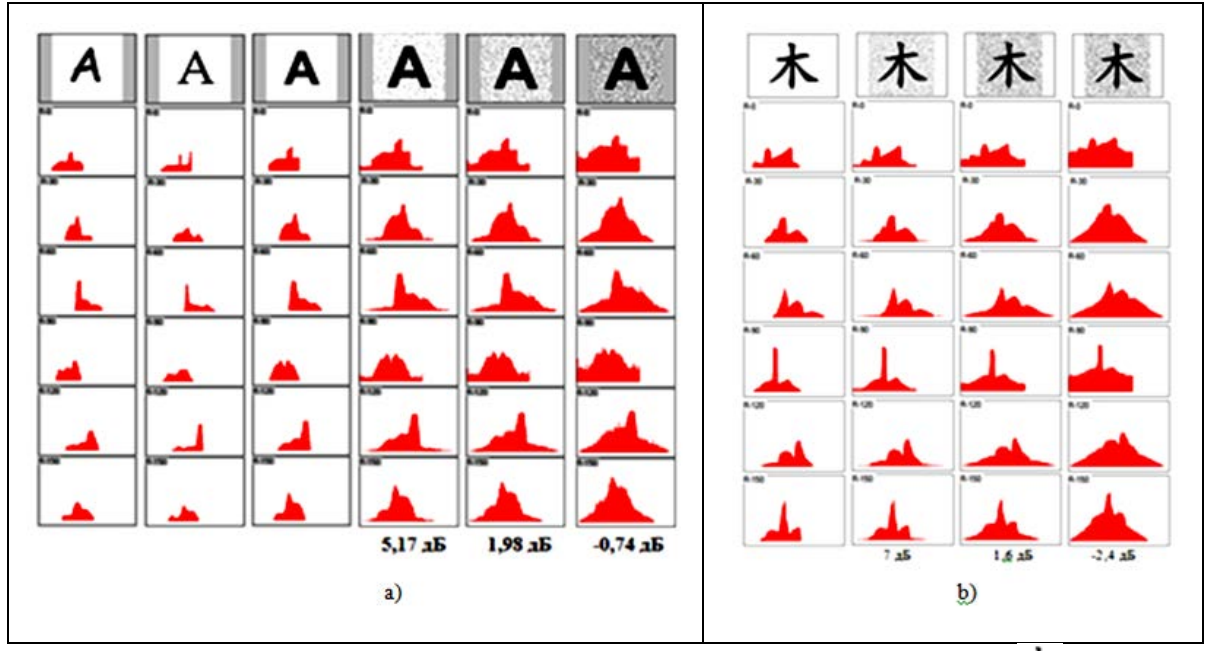

Figure 4: RT for different images letters "A" and the hieroglyph 木

Confident recognition of characters is possible in signal / noise ratio up to level $2 \mathrm{~dB}$. We should also mention the peculiarity of RT for finding of straight lines and segments in the image (fig. 4, b). And lines, coinciding with the direction of the slope of the RT, are clearly detected even at signal / noise ratio to $-5 \mathrm{~dB}$ (fig. $4, \mathrm{~b}$ ).

\section{Impact of the image rotation on the result of identification based on characteristic feature as RT projections}

Since $\varphi$ - is the angle between the direction of building RT of projection and direction of the coordinate system where there is an image, it does not matter which of these systems is fixed and which rotates. As $\varphi=30^{\circ}$ is not rotated but for normally oriented image $\left(0^{\circ}\right)$ of the construction of the RT in the direction of $30^{\circ}$, as well as $\varphi=30^{\circ}$ for reverse other situation - for the image at the angle of $30^{\circ}$ in the construction of RT in the direction $0^{\circ}$ (In both these cases, the RT formula will look.

$$
\check{f}_{30}(p, 30)=R_{30} f=\int_{-\infty}^{\infty} f\left(\frac{\sqrt{3}}{2} p-\frac{1}{2} s, \frac{1}{2} p+\frac{\sqrt{3}}{2} s\right) d s .
$$

On the basis of mentioned above CAHS it can be illustrated by constructing of RT projections of the letter "A», which is rotated on $0^{\circ}, 5^{\circ}, 10^{\circ}, 15^{\circ}, 20^{\circ}, 25^{\circ}$ and $30^{\circ}$ (fig. 5), and where for each image a graphical interpretation of the corresponding transformations is shown. 
As can be seen from Fig. 5 - RT projection that is rotated on the $30^{\circ}$ letter " $A$ " in the direction $0^{\circ}$ repeats completely the RT projection of not rotated letter "A" $\left(0^{\circ}\right)$ in the direction $30^{\circ}$.

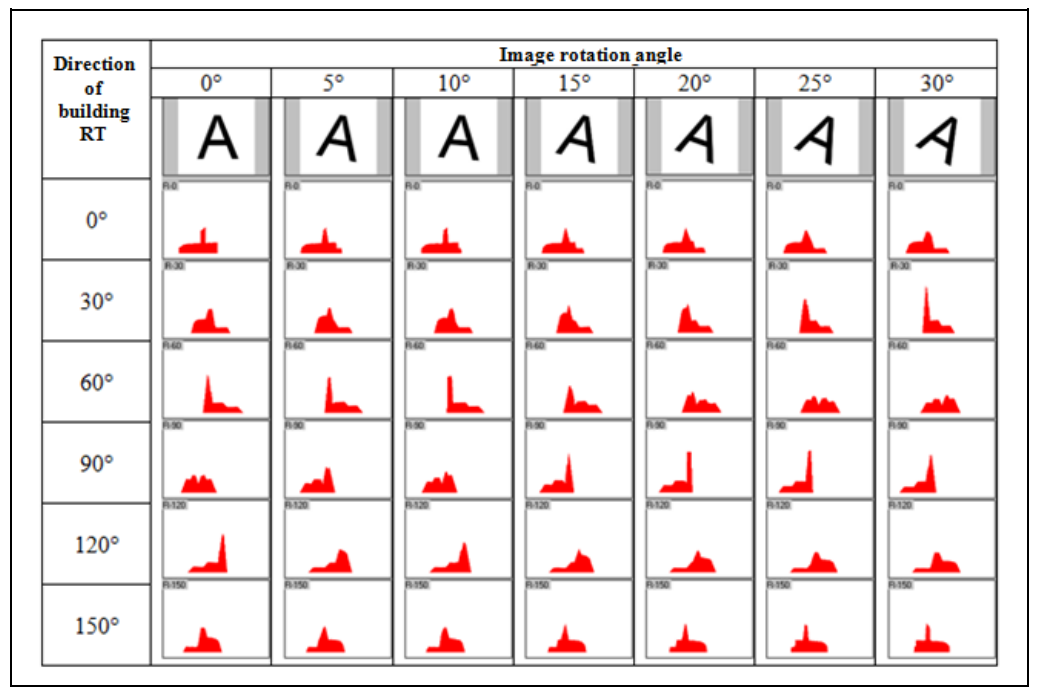

Figure 5: Example implementation of RT for characters with different angles of rotation

Obviously, this regularity is true for any other angles, multiple constructing to RT projections of step. Since the synthesized CAHS selects six projections on RT ranging from $0^{\circ}$ to $\pi$ (RT projection of range from $\pi$ to $2 \pi$ will be mirror images of the projections in the directions, which are an extension of directions of range from $0^{\circ}$ to $\pi$ ), then step (discrete) of projections constructing is $\pi / 6=30^{\circ}$. Thus, selected characteristic features are not sensitive to the rotation of the input angle of image multiple to $30^{\circ}$.

Research has shown that, when it is necessary, to select a larger number RT projections by hardware we can use two or more similar CAHS, which are rotated relative to each other by the angle $\beta$ (3) along an axis perpendicular to their plane.

For the case of two CAHS on formula (3) $\angle \beta=15^{\circ}$, we get 12 directions in increments $15^{\circ}$ (fig. 6). Fig. 6 indicates by the solid arrows the direction of the first cell medium (CM), is dashes - direction of second $\mathrm{CM}$, which is rotated by an angle relative to the first $15^{\circ}$.

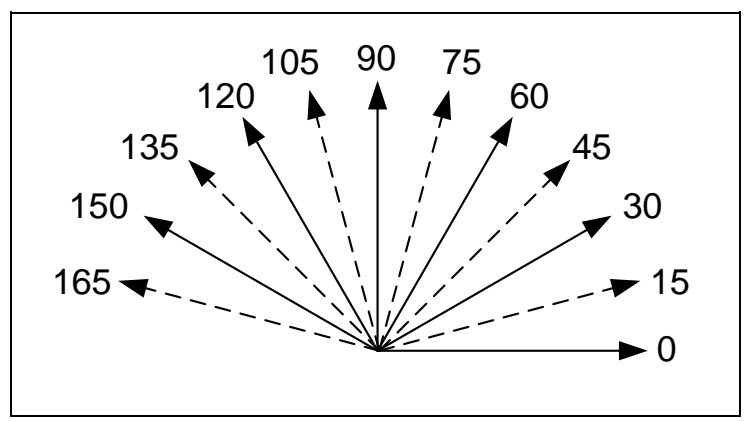

Figure 6: Directions of two CAHS rotated relative to each other on $15^{\circ}$ 
Thus, multilayer CAHS, which directions are shown in fig. 6 is insensitive to the rotation of the input image on the angle, which multiple to $15^{\circ}$.

The conducted studies has shown, that rotation of the input image at an angle from $2^{\circ}$ to $4^{\circ}$ has almost no influence on the result of the numbers identification based on characteristic feature as RT projections. The fig. 7 shows examples of RT projections of digit "5", which is normally oriented (fig. 7a) is rotated by an angle of $2^{\circ}$ (fig. $7, \mathrm{~b}$ ) and rotated by an angle of $4^{\circ}$ (fig. 7, c). As can be seen from Fig. 7, almost all projections of the direction with the same name are the same.

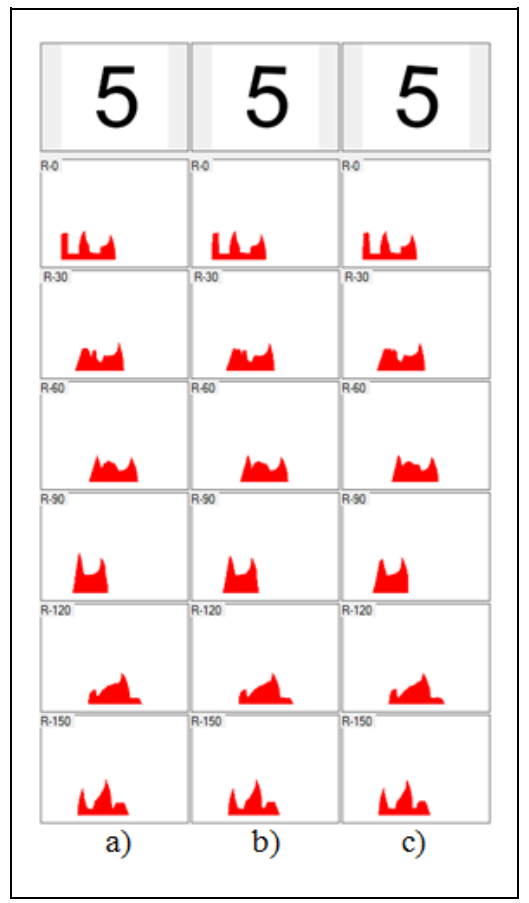

Figure 7: RT projections for the image of digit " 5 " (a) of rotating on $2^{\circ}$ and $4^{\circ}(\mathrm{b}, \mathrm{c})$

Taking into account the fact that at rotation of input image a gradual transition of one of selected projection from one discrete value to another (for example in Fig. 5 - by lines), it is possible to use not all full set of CAHS, but the half of the amount with the addition of missed projections with calculated values based on the approximation of neighboring units. In our case for the implementation of constructing of RT projections with increments of $3^{\circ}$ (as a middle value between $2^{\circ}$ and $4^{\circ}$ ) according to (3) it is necessary to use 10 of CAHS. But for the application of the approximating functions it is enough to use CAHS with just 5 layers: hardware will be allocate with the projections by directions $0^{\circ}, 6^{\circ}, 12^{\circ}$, etc. By approximation we obtain intermediate values $3^{\circ}, 9^{\circ}, 15^{\circ}$, etc. Thus, such use of the approximation allows to increase the number of allocated projections, while reducing the hardware costs.

The approach based on the use of multilayer CAHS and approximations of the original data allows you to build devices for hardware allocation based on characteristic features of the Radon transform, which is insensitive (invariant) to the rotation of the input image. 


\section{RT Properties under Scaling and Shifting of the Input Image}

It is known, that the Radon transform has the shift and scaling properties [1-3]. The offset function in the spatial domain leads to a displacement of projection along the $p$, and its magnitude depends on the angle $\varphi$ (fig. 2). This is illustrated in fig. 8, where examples of RT for image of the letter " $A$ " are shifted in the directions $0^{\circ}, 45^{\circ}$ and $90^{\circ}$ are shown: value and shape of the projections of the same name are the same, except their displacement. If the direction shift of the input image and built RT projection coincided, the resulting projection did not move at all (fig. 8 a) and b) the projection of R-0 for directions $0^{\circ}$, and a), d) the projection of R-90 for directions $90^{\circ}$ ) and most shifted projection that has the perpendicular direction (fig. 8a) and d) of the projection $\mathrm{R}-0$, and $\mathrm{a}$ ), b) the projection of R-90), which fully confirms the dependence of the displacement of the angle $\varphi$.

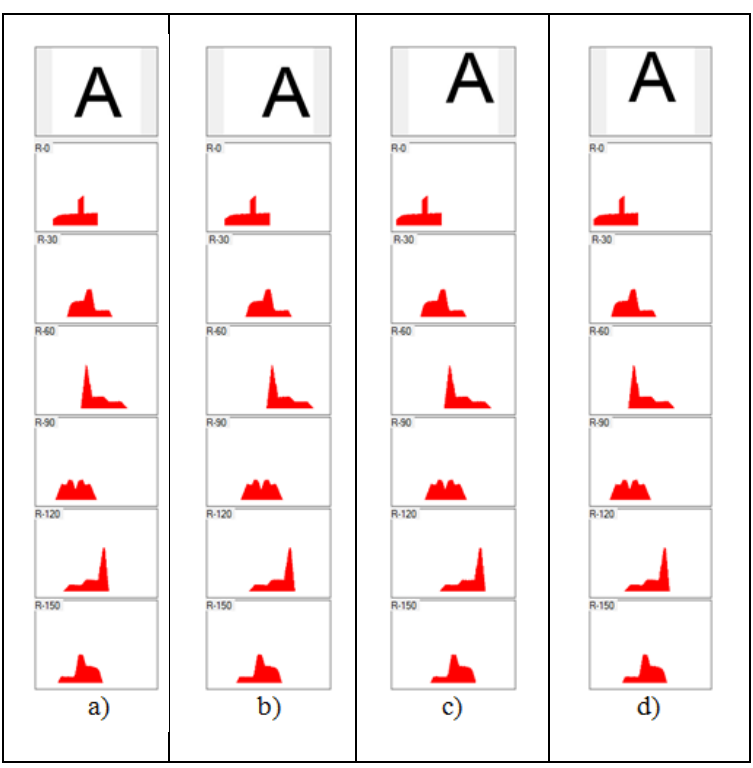

Figure 8: RT projection for image of letters «A» (a) at the shift of direction of the input image $0^{\circ}(\mathrm{b}), 45^{\circ}$ (c) and $90^{\circ}$ (d)

Similarly to the scaling function in the spatial domain scaling of projections is undergone, but its value does not depend on the angle $\varphi$, and completely repeats scaling factor $\mathrm{m}$ of the function itself.

In fig. 9 we show examples of projections of RT for input image of letters «A» (a), which is scaled by proportional with coefficient to $m=1,5$ by the two coordinates (b), scaled only in the $x$-axis (direction CAHS $0^{\circ}$ ) with coefficients $m=1,25$ and $m=1,5$ (c, d), and the $y$-axis only (direction CAHS $90^{\circ}$ ) with coefficients $m=1,25$ and $m=1,5(\mathrm{e}, \mathrm{f})$. 


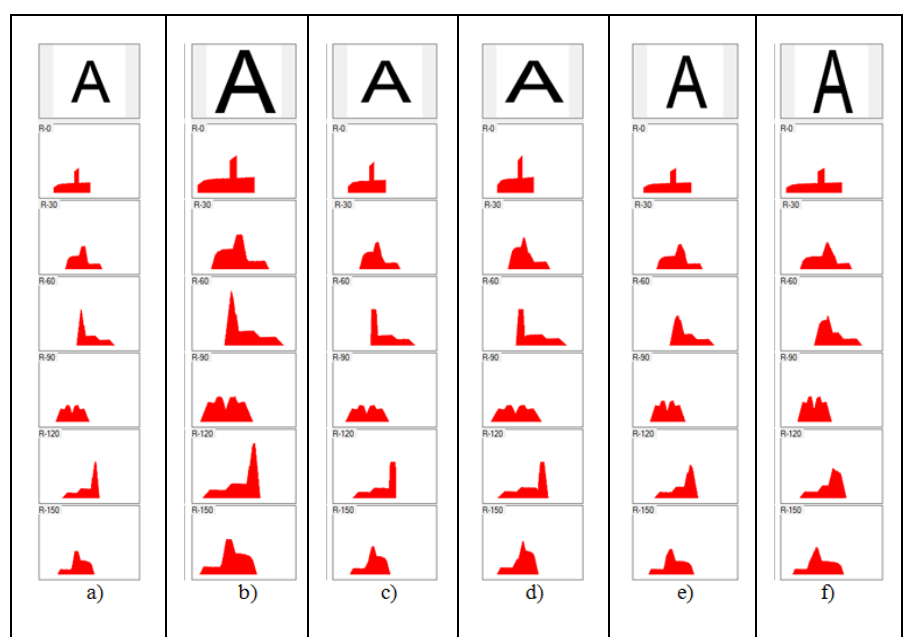

Figure 8: RT projection for image of letters «A» (a) at the shift of direction of the input image $0^{\circ}$ (b), $45^{\circ}$ (c) and $90^{\circ}(\mathrm{d})$

As can be seen from Figure. 9 a) and b) - scaling obtained projections of RT repeats completely the proportional scaling of the input image. Thus the sizes of form of the built projections also vary proportionally to $m$.

More difficult are the special cases of non-proportional scaling along one of the coordinates (fig. 9, c) , d) , e) , f)), where not only disproportionate strain derived projections are observed, as well as some changes of their shape. For non-proportional scaling of function in the spatial domain of constructed projection is again dependable as in the case of the displacement from angle $\varphi$. So, if the direction of non-proportional scaling of the input image and build of RT projection coincide, then the obtained sinogram (fig. 9, a) and c), d) of projection R-0; a) и e), f) of projection R-90) will have the same width at extended to $\mathrm{m}$ times in the absolute values. While sinogram of perpendicular direction (fig. 9, a) and c), d) of projection R-90; a) and e), f) of projection $\mathrm{R}-0$ ) will have an opposite pattern in form of a larger in $\mathrm{m}$ time width and equal to absolute values. Projections for the rest of the intermediate directions will be deformed in both the width and the absolute values on the amount from 1 to $\mathrm{m}$ and will depend on the angle $\varphi$. This will be accompanied by some changes in the form, which is explained by the disproportionate change of thickness and inclination angles of elements of the input image at a disproportionate scaled, which generally can be regarded as a kind of deformation or distortion.

Characteristic features in the form of RT projections are invariant to shifts and scaling of the input image. And they also possess resistance to deformation and distortion in the form of nonproportional scaling in one of direction on condition of account of the specification of these cases during operation of comparing of the CF with standard.

\section{Conclusion}


Stepan Bilan, Ruslan Motornyuk and Sergii Bilan; Method of Hardware Selection of Characteristic Features Based on Radon Transformation and not Sensitive to Rotation, Shifting and Scale of the Input Images, Advances in Image and Video Processing, Volume 2 No 4, Aug (2014); pp: 12-23

The developed method allows to perform by hardware the selection of characteristic features based on Radon transform, which are insensitive to changes in the input image such as rotation, shift, zoom (including disproportional that is performed in one direction). Moreover, the invariance rotation is ensured by increasing of the number of layers and CAHS approximation of the original data, and the shift and scaling - only through considering when compared with standard characteristics of value of the shift and scaling factor of the input image.

\section{REFERENCES}

[1]. Radon J. K. A., Uber die Bestimmung von Funktionen durch ihre Integralwerte langs gewisser Mannigfaltigkeiten, Berichte Sachsische Akademie der Wissenschaften, Leipzig, Mathematisch-Physikalische Klasse, 1917. 69: p. 262-277.

[2]. Toft P., The Radon Transform: Theory and Implementation. PhD thesis, Dept. of Math. Modelling Section for Digital Signal Processing, Technical Univ. of Denmark, 1996. p. 326.

[3]. Левин Г.Г., Вишняков Г.Н., “Оптическая томография”, Радио и связь, 1989. р. 224.

[4]. Грузман И.С., “Математические задачи компьютерной томографии”. Соросовский образовательный журнал, 2001. 7(5).

[5]. Отдыхов Р.Х., Вершок Д.А., Алгоритм выделения информативных признаков на основе преобразования Радона в системе распознавания рукописных признаков. Известия НАН Беларуси. Сер. физ.-техн. Наук, 1998. (3): р. 103-107.

[6]. Варанов В.Г., Храмов А.Г., Дискретное веерное преобразование Радона в задаче выделения центров ветвей сетчатых структур. Институт систем обработки изображений РАН "Компьютерная оптика", 2002. (23): p. 44-47.

[7]. Кобасяр М.І., Русин В. П., Детектування кривих з бінарних зображень за допомогою перетворення Радона. Вісник Національного університету “Львівська політехніка" “Радіоелектроніка та телекомунікації", 2001. (428): р. 6-9.

[8]. Волегов Д. В., Гусев В. В., Юрин Д. В., Обнаружение прямых линий на изображениях на основе преобразования Хартли. Быстрое преобразование Хафа. 16-я международная конференция по компьютерной графике и ее приложениям, (ГрафиКон2006), Россия, Новосибирск, Академгородок, 2006. с. $182-191$.

[9]. Kadyrov A., Petrou M., The Trace Transform and Its Applications. IEEE Ransactions On Pattern Analysis And Machine Intelligence, 2001. 23(8): p. 811-828.

[10]. Von Neumann J., Theory of Self-Reproducing Automata: Edited and completed by A. Burks., University of Illinois Press, 1966. 
[11]. Zuse K., Calculating Space. Translated from German. - Tech. Transl. AZT-70-64- GEMIT. - MIT Project MAC, 1970.

[12]. Ulam S., Random Processes and Transformations. Procedings Int. Congr. Mathem, 1952. (2): p. 264-275.

[13]. Blanchard F., et al., Topological and Measure-Theoretic Properties of One-Dimensional Cellular Automata. Physica D: Nonlinear Phenomena, 1997. 103(1-4): p. 86-99.

[14]. Belan S., Specialized cellular structures for image contour analysis, Cybernetics and Systems Analysis, 2011. 47(5): p. 695-704.

[15]. Belan S., and Motornyuk R., Extraction of characteristic features of images with the help of the radon transform and its hardware implementation in terms of cellular automata. Cybernetics and Systems Analysis, 2013. 49(1): p. 7-14.

[16]. Wolfram S., Cellular Automata. Los Alamos Science, 1983. 9: p. 2-21.

[17]. Nicoladie D. Tam., Hexagonal pixel-array for efficient spatial computation for motion-detection preprocessing of visual scenes, Advances in image and video processing, 2014. 2(2): p. 26-36.

[18]. Wolfram S., Random Sequence Generation by Cellular Automata. Advances in Applied Mathematics, 1986. 7: p. $429-432$. 